Versuche ist demnach: die Bildung geringer, aber deutlicb nachweisbarer Mengen von $\mathrm{NO}_{2} \mathrm{NH}_{4}$ bei Verbrennung von vollkommen gereinigtem $H$ in voilkommen gereinigter Luft.

W ien, den 1. December 1877.

(Chem. Lab. d. k. k. Hochschale f. Bodencaltur.)

550. R. Ni itzki: Ueber Nitranilsäure.

(Vorláufige Notiz.)

(Eingegangen am 13. December.)

In der Hoffnung Diazo- oder Nitrosoverbindungen $z u$ erbalten, leitete ich salpetrige Säure in eine mit Eis gekühlte Lösung von Hydrochinon. Die erste Erscheinung, welche dabei auftrat, war, dass die ganze Flüssigkeit zu einem Brei von Chinhydronkrystallen erstarrte, welche nach einiger Zeit wieder verschwanden.

Das Hydrochinon wurde offenbar fürs erste $z a$ Chinon oxydirt.

Setzte man das Einleiten fort, bis das Gas nicht mehr absorbirt wurde, so hatten sich am Boden des Gefässes kleine, goldgelbe Krystallnadeln ausgeschieden.

Ein Zufall belehrte mich, dass diese Ausscheidung sich bedeutend vermehrt, wenn man zum Schluss einige Tropfen Wasser hinzufügt. Der erhaltene Körper wurde mit Aether abgewaschen in dem er fast unlöslich ist.

In Wasser löst er sich mit grosser Leichtigkeit. Fügt man zu dieser Lösung etwas Kalilauge, so scheiden sich sofort kleine, schwefelgelbe Krystallnadeln einer sehr schwer löslichen Kaliumverbindung aus.

Durch Umkrystallisiren aus heissem Wasser konnte dieses Salz in schönen, bellgelben Nadeln erhalten werden, welche nach dem Trocknen einen blauen Flächenschimmer zeigen. Die Verbindung löst sich in kaltem Wasser kaum leichter als Kaliumplatinchlorid, ziemlich reichlich in beissem Wasser. In der kaltgesättigten Lösung bewirkt Kalilauge eine Abscheidung von feinen, schilleraden Nadeln. Trocken erhitzt verpufft der Körper ziemlich lebhaft. Die Analyse ergab einen so niedrigen Wasserstoffgehalt, dass derselbe auf Rechnung des üblichen analytischen Fehlers geschrieben werden muss. Aus derselben berechuet sich die Formel $\mathrm{C}_{6} \mathrm{~N}_{2} \mathrm{O}_{8} \mathrm{~K}_{2}$.

Theorie.

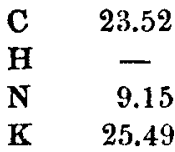

Versuch.

23.85

0.48

23.83

0.27

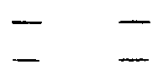

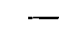

9.59 
Da der Körper alle Eigenschaften eines Nitroderivates hat. so ist nicht daran zu zweifeln, dass die beiden Stickstoffatowe 2 Nitrogruppen repräsentiren.

Von der Chinonformel ausgebend gelangt man alsdann zu der Formel $\mathrm{C}_{6}\left(\mathrm{~N} \mathrm{O}_{2}\right)_{2}(\mathrm{KO})_{2} \mathrm{O}_{2}$.

Der Körper ist demnach das Kaliumsalz eines DinitrodioxyChinons, welches ich, da es der Chloranilsãure durchans analog ist, Nitranilsäure nennen will.

Ueber die Bildung der Substanz ist kaum ein $Z_{\text {weifel. Offenbar }}$ wird hier zuerst Tetranitrochinon (Nitranil) gebildet, welches unter dem Einflusse des Kalihydrats, oder auch schon deg Wassers, zwei Nitrogruppen unter Bildung von $2 \mathrm{HN} \mathrm{O}_{2}$ gegen Hydroxyle austauscht.

Ich habe Grund anzunehmen, dass diese Umsetzung schon durch Wasser bewirkt wirkt und dass das Nitranil selbst sebr unbeständig ist. Schon der Umstand, dass der Körper durch Wasserzusatz aus der àtherischen Lōsung ausgeschieden wird und bei dieser Ausscbeidung reichlich rothe Dämpfe auftreten, spricht dafür.

Löst man die ausgeschiedene Substanz in Wasser und lässt über Schwefelsäure verdunsten, so erhält man scbön goldgelbe Prismen mit b]äulichem Dicbroïsmus.

Obwohl ich diesen Körper noch nicht in zur Analyse geeigueter Quantität und Reinbeit hatte, da er sich wegen seiner leichten Löslichkeit nur mit grossem Verlust umkrystallisiren lässt, geht ans seinem ganzen Verhalten hervor, dass hier Nitranilsäure und kein Nitranil vorliegt. Er bewirkt nämlich schon in Cblorkaliumlösung einen Niederschlag der erwähnten Kahumverbindung, dabei ist aber ebensowenig wie bei der Behandlung mit Kalihydrat die Bildung von salpetriger Säure nachzuweisen.

Die Nitranilsäure krystallisirt in centimeterlangen Krystallen, welche wasserbaltig sind und beim vorsichtigen Erwärmen verwittern. Im Capillarröhrchen schmelzen sie etwas über $100^{\circ} \mathrm{im}$ Krystallwaseer. Wrsser lost sie sehr leicht, Alkobol entzieht ihnen das Krystallwasser, whne sie merklich zu lösen.

Die wasserfreie Substanz verpufft gegen $170^{\circ}$, ohne vorher 7.11 schmelzen.

Die Nitranilsäure besitzt einen stark ausgesprochenen Säurecharakter; sie schmeckt nicht bitter, wie die meisten Nitroderivate, sondern stark sauer, nebenbei eisenartig adstringirend. Mit den meisten Metallen bildet sie sehr schw - b̈sliche, schön krystallisirende Salze und treibt dahei sogar die Mineralsäuren aus. So bewirkt sie in Chlorbarium-, Chlorcalcium- und Silbernitratlösung sofort einen krystallinischen Niederschlag der entsprechenden Metallverbindungen.

Neben der Nitranilsäure scheinen noch andere Nitroderivate des 
Chinons in obigem Process gebildet zu werden, ein grosser Theil des Hydrochinons wird jedoch stets in Oxalsăure übergeführt.

Ich gedenke zunächst das Verhalten der Nitranilsäure gegen reducirende Agentien zu studiren. Da die Beschaffung des Materials jedoch noch erhebliche Schwierigkeiten bietet, hoffe ich mir durch dieze Mittheilung die weitere Bearbeitung dieses Gebietes zu sichern.

Leiden, Universităts-Laboratorium.

\section{O. Hesse: Beitrag zur Kenntniss des von Henry und Delondre Chinidin genannten Alkaloïds.}

(Eingeganged am 21. December.)

Anlässlich der Bebauptung von Pasteur, sein Chinidin sei mit dem Alkaloïd identisch, welches Henry und Delondre 1833 aus einer gelben Chinarinde abschieden und ebenfalls Chinidin nannten, untersuchten Bouchardat und Boudet') das Letztere und zwar das Sulfat desselben, wobei sie fanden, dass dasselbe in anderer Weise auf das polarisirte Licht reagire, als $\mathrm{Pasteur}$ von seinem Alkaloïd angegeben batte. Obwohl Bouchardat und Boudet diese Substanz nicht weiter prüften, ob dieselbe einheitlich oder ein Gemisch sei, so berechtigt doch das bei der optischen Probe erbaltene Resultat zu der Annahme, dass beide Alkaloide ganz bestimmt von einander verschieden seien. Dass fragliches Sulfat etwas anderes sei, als für das es ausgegeben wurde, dürfte in Anbetracht des Zweckes jener Untersuchung kaum anzunebmen sein.

Neuerdinga bat jedoch $\mathrm{Bouchardat}{ }^{2}$ ) der früheren Angabe eine neue Lesart untergeschoben, indem er nämlich die Bezeichnung des betreffenden Präparates: ${ }_{n}$ Chiuidinsulfat von Henry und Delondre" verschwinden liess und dafür die Bezeichnung ${ }^{\circ} \mathrm{Chinidin-}$ sulfat von Delondre" gebrauchte. Ausserdem behauptet Bouchardat, Delondre babe lange Zeit bindurch das Sulfat von Winckler's Alkaloid Chinidinsulfat genannt.

Bei dem Interesse, weiches ich an der richtigen Wiedergabe der Geschichte des fraglichen Gegenstandes habe, glaubte ich daher den Gegenstand von nenem untersuchen za sollen. Ich wende mich deshalb an Delondre selbst.

Delondre sagt nämlich in seiner Quinologie, welche er 1854 in Gemeinschaft mit Bouchardat herausgab, dass unter Chinidin ein Alkaloid zu verstehen sei, welches früher Henry und Delondre für ein besonderes Hydrat des Chinins gehalten bätten. Es

8) Moniteur scientifique (8) 7, 602 . 